\title{
PRIMITIVE SPACES OF MATRICES OF BOUNDED RANK.II
}

\author{
M. D. ATKINSON \\ (Received 18 December 1981) \\ Communicated by D. E. Taylor
}

\begin{abstract}
The classification of spaces of matrices of bounded rank is known to depend upon 'primitive' spaces, whose structure is considerably restricted. A characterisation of an infinite class of primitive spaces is given. The result is then applied to obtain a complete description of spaces whose matrices have rank at most 3 .
\end{abstract}

1980 Mathematics subject classification (Amer. Math. Soc.): 15 A 30, 15 A 03.

\section{Introduction}

Given several arbitrary $m \times n$ matrices of rank at most $r<\min (m, n)$ it is 'likely' that there will be a linear combination of them having rank greater than $r$. In other words, the hypothesis that every matrix in a vector space of matrices has rank at most $r$, is a restrictive one. However there are enough examples of spaces of matrices which have this property to make the classification of such spaces rather difficult. To make some progress on this problem Atkinson and Lloyd (1981) introduced the idea of a primitive space and showed that it was sufficient to classify the primitive spaces. This paper takes a step towards this classification by giving a simple characterisation of an infinite class of primitive spaces. The result is strong enough to lead to a complete description of primitive spaces (and hence all spaces) whose matrices have rank at most 3.

This work was carried out at Carleton University, Ottawa and partly supported by the Canadian National Science and Energy Research Council under Grant No. A7171.

(1) Copyright Australian Mathematical Society 1983 
We briefly review the necessary definitions. Two spaces $\mathcal{X}, \mathscr{Y}$ of $m \times n$ matrices are said to be equivalent if

$$
\mathcal{X}=P \mathscr{Y} Q=\{P Y Q: Y \in \mathcal{Y}\}
$$

for some non-singular $m \times m$ and $n \times n$ matrices $P$ and $Q$. This definition recognises that $\mathcal{X}$ and $\mathscr{Y}$ commonly arise as the matrix representations of spaces of linear transformations from an $m$-dimensional vector space $U$ into a $n$-dimensional vector space $V ; \mathfrak{X}$ is equivalent to $\mathcal{Y}$ if $\mathscr{X}$ represents the same transformations as $\mathcal{O}$ but with respect to different bases of $U$ and $V$.

We denote the maximum of the ranks of the matrices (or transformations) in a space $\mathcal{X}$ by $\rho(X)$. It is shown in Atkinson and Lloyd (1980) that, when the ground field has more than $\rho(\mathscr{X})$ elements, $\rho(\mathscr{X})$ cannot increase if the field is extended. For this reason we shall always assume that the cardinal of the ground field is greater than the rank of any matrix in the spaces under consideration.

A space $\mathcal{X}$ of matrices is defined to be primitive if the following conditions hold:

(i) no space equivalent to $\mathscr{X}$ consists of matrices with a fixed row equal to zero,

(ii) no space equivalent to $\mathcal{X}$ consists of matrices with a fixed column equal to zero,

(iii) no space equivalent to $\mathscr{X}$ consists of matrices $[u Y]$ where $u$ is a column vector and rank $Y<\rho(\mathscr{X})$,

(iv) no space equivalent to $\mathcal{X}$ consists of matrices $\left[\begin{array}{l}u \\ Y\end{array}\right]$ where $u$ is a row vector and rank $Y<\rho(\mathscr{X})$.

Translating these conditions into the language of linear transformations we can define a space $\mathcal{Z}$ of linear transformations from $U$ into $V$ to be primitive if

(i) the images of $U$ under the transformations in $Z$ generate $V$,

(ii) no non-zero element of $U$ is in the kernel of every transformation of $\mathscr{Z}$,

(iii) $U$ has no subspace $U_{1}$ of codimension 1 such that $\mathscr{Z}$ restricted to $U_{1}$ consists of transformations of rank at most $\rho(\mathscr{Z})-1$,

(iv) $V$ has no 1-dimensional subspace $V_{1}$ such that all the composite transformations $U \stackrel{z}{\rightarrow} V \stackrel{\pi}{\rightarrow} V / V_{1}, z \in \mathscr{Z}$ and $\pi$ the canonical projection, have rank at most $\rho(\mathscr{Z})-1$.

It is not difficult to see that conditions (iii) and (iv) are equivalent (respectively) to

(iii) $U=\langle\operatorname{ker} z: z \in \mathscr{Z}$ and rank $z=\rho(\mathscr{L})\rangle$ and (iv) $0=\cap\{z: z \in \mathscr{Z}$ and $\operatorname{rank} z=\rho(\mathscr{Z})\}$.

The main justification for the idea of primitivity is the following result of Atkinson and Lloyd (1981).

THEOREM A. If $X$ is a space of $m \times n$ matrices then there exists a primitive space $\mathcal{Y}$ and integers $p, q \geqslant 0$ with $\rho(\mathscr{X})=p+q+\rho(\mathscr{Y})$ such that $\mathscr{X}$ is equivalent to a 
space of matrices of the form

$$
\left[\begin{array}{c|cc}
p \times q & & \\
\hline & Y & 0 \\
& 0 & 0
\end{array}\right]
$$

where the submatrices $Y$ constitute the primitive space $\mathcal{Y}$.

Moreover for a primitive space $\mathfrak{X}(p=0, q=0, \mathfrak{X}=\mathcal{Y})$ with $r=\rho(\mathfrak{X})$ one of the following occurs:

(i) $m=r+1, n \leqslant \frac{1}{2} r(r+1)$,

(ii) $m \leqslant \frac{1}{2} r(r+1), n=r+1$,

(iii) for some integers $c, d \geqslant 2$ with $c+d=r$,

$m \leqslant c+1+\frac{1}{2} d(d+1)$ and $n \leqslant d+1+\frac{1}{2} c(c+1)$.

Note particularly that in a primitive space $\mathfrak{X}$ of $m \times n$ matrices with $\rho(\mathcal{X})=r$ we have $m, n \leqslant \frac{1}{2} r(r+1)$. Atkinson and Lloyd (1981) gave examples to demonstrate that this bound is tight (although for $r \geqslant 3$ it cannot be attained simultaneously by $m$ and $n$ ). In the next section we generalise these examples and sketch a construction which shows that, for fixed $m, n$ and $r$, there may be an infinity of inequivalent primitive spaces of $m \times n$ matrices. In Section 3 we prove that the primitive spaces constructed in Section 2 are the only ones which can arise when $m$ or $n$ is close to the upper bound $\frac{1}{2} r(r+1)$. In the last section we obtain a description of all primitive spaces whose matrices have rank at most 3 . The precise determination actually depends upon the ground field and we give full details only in the algebraically closed case. Finally we observe that our results have implications for the stronger hypothesis, studied by Westwick (1972), of a space all of whose non-zero matrices have rank exactly 2 (or rank exactly 3 ).

\section{Infinitely many primitive spaces}

Throughout this section $U$ and $V$ denote vector spaces and $\operatorname{dim} U=r+1$. If $\phi$ : $U \times U \rightarrow V$ is any alternating bilinear mapping we shall let $S(\phi)$ denote the space of all linear transformations $\phi_{u}, u \in U$, where

$$
\phi_{u}(x)=\phi(u, x) \text { for all } x \in U .
$$

Since $\phi_{u}(u)=0$ we have rank $\phi_{u} \leqslant r$. In general however $S(\phi)$ need not contain any transformations of rank precisely $r$, nor need $S(\phi)$ be primitive.

Let $L(\phi)=U \oplus V$ and define a multiplication on $L(\phi)$ by the rule

$$
(u+v)\left(u^{\prime}+v^{\prime}\right)=\phi\left(u, u^{\prime}\right)
$$


Endowed with this multiplication $L(\phi)$ is a Lie algebra of nilpotency class 2 and we are in the situation covered by the proposition in Section 2 of Vaughan-Lee (1974). Interpreting this proposition in our notation (and checking that its proof is independent of the ground field) we obtain

LEMma 1. Suppose that $\{u: \phi(u, U)$ has dimension $r\}$ does not generate $U$. Then $U$ has a proper subspace $U_{1}$ such that $\left\langle\phi\left(U_{1}, U_{1}\right)\right\rangle=\langle\phi(U, U)\rangle$.

In order to apply this lemma we specialize even further. From now on we take $\operatorname{dim} V>1+\frac{1}{2} r(r-1)$ and consider only alternating bilinear mappings $\phi$ such that $V=\langle\phi(U, U)\rangle$. Under these conditions we have

LEMMA 2. $\rho(S(\phi))=r$ and $S(\phi)$ is primitive.

Proof. Notice first that $\{u: \phi(u, U)$ has dimension $r\}$ generates $U$; for otherwise the previous lemma would show that $V=\left\langle\phi\left(U_{1}, U_{1}\right)\right\rangle$ for some subspace $U_{1}$ of $U$ of dimension at most $r$, and so we would obtain $\operatorname{dim} V \leqslant \frac{1}{2} r(r-1)$. In particular $S(\phi)$ contains transformations of rank $r$ and therefore $\rho(S(\phi))=r$.

Checking the requirements for primitivity we have condition (i) immediately from $V=\langle\phi(U, U)\rangle$. Condition (ii) is also easy for if it did not hold there would be a non-zero vector $u \in U$ such that $\phi(u, U)=0$ and it would follow that $\operatorname{dim} V=\operatorname{dim}(\langle\phi(U, U)\rangle) \leqslant \frac{1}{2} r(r-1)$.

The initial observation of this proof can be rephrased as

$$
\left\{\operatorname{ker} \phi_{u}: \phi_{u} \text { has rank } r\right\} \text { generates } U
$$

so requirement (iii)' holds. Finally suppose $V$ has a 1 -dimensional subspace $V_{1}$, such that all the compositions

$$
U \stackrel{\phi_{u}}{\rightarrow} V \stackrel{\pi}{\rightarrow} V / V_{1}
$$

have rank at most $r-1$. Applying Lemma 1 to the bilinear mapping $\pi \phi$ shows that $\operatorname{dim} V / V_{1} \leqslant \frac{1}{2} r(r-1)$ and hence $\operatorname{dim} V \leqslant 1+\frac{1}{2} r(r-1)$. This contradiction proves that there can be no such subspace $V_{1}$, and so requirement (iv) holds.

By arguing along the lines followed by Atkinson and Westwick (to appear) one can show without much difficulty that, with two alternating bilinear mappings $\phi_{1}$ and $\phi_{2}$ subject to the conditions above, we have $S\left(\phi_{1}\right)$ equivalent to $S\left(\phi_{2}\right)$ if and only if the Lie algebras $L\left(\phi_{1}\right)$ and $L\left(\phi_{2}\right)$ are isomorphic. Appealing to the results of Gauger (1973), especially Section 2 and Theorem 7.8, we can obtain (over an 
algebraically closed field) infinitely many inequivalent primitive spaces $S(\phi)$ provided that $n=\operatorname{dim} V$ satisfies

$$
\begin{gathered}
1+\frac{1}{2} r(r-1)<n<\frac{1}{2} r(r+1) \quad \text { and } \\
(r+1)^{2}<\left[\frac{1}{2} r(r+1)-n\right] n+1 .
\end{gathered}
$$

\section{Classification}

In the previous section it was shown that, if $\operatorname{dim} U=r+1, \operatorname{dim} V>1+$ $\frac{1}{2} r(r-1)$ and $\phi: U \times U \rightarrow V$ is an alternating bilinear mapping whose image spans $V$, then $S(\phi)$ is a primitive space. In this section we prove the following strong converse.

THEOREM B. Let $\mathfrak{X}$ be a primitive space of $m \times n$ matrices with $\rho(\mathscr{X})=r$ and $n>1+\frac{1}{2} r(r-1)$. Then $m=r+1=\operatorname{dim} \mathcal{X}$ and $\mathcal{X}$ is a matrix representation of a space $S(\phi)$ for some alternating bilinear mapping defined on an $(r+1)$-dimensional vector space.

The key to this theorem is a lemma which may have implications in other investigations of this type.

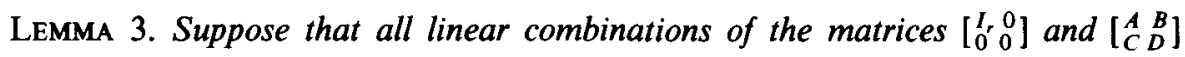
have rank at most $r$.

Then $D=0$ and $C A^{k} B=0, k=0,1,2, \ldots$

Proof. That $D=0$ and $C B=0$ was proved by Flanders (1962) but our proof would not be shortened significantly by assuming these facts. The lemma will hold provided that, for every submatrix of $\left[\begin{array}{ll}A & B \\ C & D\end{array}\right]$ of the form $\left[\begin{array}{ll}A & 0 \\ u & d\end{array}\right]$ where $u$ is a row of $C$ and $v$ is a column of $B$, we have $d=0$ and $u A^{k} v=0, k=0,1,2, \ldots$ Consequently it suffices to consider this special case.

If $\lambda$ is transcendental over the ground field the matrix

$$
\left[\begin{array}{cc}
A-\lambda I & v \\
u & d
\end{array}\right]
$$

is singular, so there exists some non-zero row $(r+1)$-vector $[x, \alpha](x$ being an $r$-vector) with the property

$$
[x, \alpha]\left[\begin{array}{cc}
A-\lambda I & v \\
u & d
\end{array}\right]=0 .
$$

Among the vectors which satisfy this equation we choose one whose components are polynomials in $\lambda$ of least degree $\varepsilon \leqslant r$. We may put $x=x_{0}+\lambda x_{1}+\cdots+\lambda^{\varepsilon} x_{\varepsilon}$ 
and $\alpha=\alpha_{0}+\lambda \alpha_{1}+\cdots+\lambda^{\varepsilon} \alpha_{e}$ where each $x_{i}$ is an $r$-vector and each $\alpha_{i}$ is a scalar. Substituting in the equations

$$
x(A-\lambda I)+\alpha u=0 \text { and } x v+\alpha d=0
$$

and equating powers of $\lambda$ to zero we obtain

$$
\begin{aligned}
x_{0} A= & -\alpha_{0} u \\
x_{1} A= & x_{0}-\alpha_{1} u \\
& \vdots \\
x_{\varepsilon} A= & x_{\varepsilon-1}-\alpha_{\varepsilon} u \\
0= & x_{\varepsilon}
\end{aligned}
$$

and

$$
\begin{aligned}
x_{0} v+\alpha_{0} d= & 0 \\
& \vdots \\
x_{\varepsilon} v+\alpha_{\varepsilon} d= & 0 .
\end{aligned}
$$

The final equations of (1) and (2) give $\alpha_{\varepsilon} d=0$. By the minimality of $\varepsilon$ and since $x_{\varepsilon}=0$ we have $\alpha_{\varepsilon} \neq 0$. Thus $d=0$ and so

$$
x_{i} v=0, \quad i=0,1, \ldots, \varepsilon .
$$

The penultimate equation in (1) together with $\alpha_{e} \neq 0$ gives $u \in\left\langle x_{\varepsilon-1}\right\rangle$. This establishes the first step of an induction in which we prove that

$$
u A^{k} \in\left\langle x_{0}, x_{1}, \ldots, x_{\varepsilon-1}\right\rangle, \quad k=0,1,2, \ldots
$$

If this holds for some fixed $k$ then

$$
\begin{aligned}
u A^{k+1} & \in\left\langle x_{0} A, x_{1} A, \ldots, x_{\varepsilon-1} A\right\rangle \subseteq\left\langle u, x_{0}, x_{1}, \ldots, x_{\varepsilon-2}\right\rangle \\
& \subseteq\left\langle x_{0}, x_{1}, \ldots, x_{\varepsilon-1}\right\rangle \text { since } u \in\left\langle x_{\varepsilon-1}\right\rangle .
\end{aligned}
$$

The lemma now follows from (3) and (4).

Proof of Theorem B. Theorem A and the inequality $n>1+\frac{1}{2} r(r-1)$ show that $m=r+1$. By hypothesis, $\mathscr{X}$ contains a matrix of rank $r$ and so, replacing $\mathscr{X}$ with an equivalent space, we may take $\left[\begin{array}{ll}I_{r} & 0 \\ 0\end{array}\right] \in \mathcal{X}$. Let $A_{1}, A_{2}, \ldots$ be a basis for $\mathcal{X}$, let $x_{1}, x_{2}, \ldots$ be independent transcendentals and put $X=\Sigma x_{i} A_{i}$, a generic matrix of rank $r$. Since every linear combination of $X=\left[\begin{array}{cc}A & B \\ u & d\end{array}\right]$ and $\left[\begin{array}{ll}I_{r} & 0 \\ 0^{\prime} & 0\end{array}\right]$ has rank at most $r$, Lemma 3 tells us that $d=0$ and $u A^{k} B=0, k=0,1,2, \ldots$

Notice next that rank $B=r-1$. Certainly rank $B \leqslant r-1$ since $u \neq 0$ and rank $X=r$. On the other hand if rank $B<r-1$ the same will be true of every specialisation $\bar{B}$ of $B$ and therefore the matrices of $\mathscr{X}$ will have the form $\left[\begin{array}{ll}\bar{A} & \bar{B} \\ \bar{u} & 0\end{array}\right]$ with rank $\bar{B}<r-1$. But, since the space of matrices $\bar{B}$ has the 'row condition' of 
Atkinson and Lloyd (1981), Lemma 6 of that paper shows that $n \leqslant r+$ $\frac{1}{2}(r-1)(r-2)=1+\frac{1}{2} r(r-1)$ which is a contradiction.

Because rank $B=r-1$ the equation $u B=0$ shows that $u$ generates the null space of $B$ (regarding $B$ as an operator defined on row $r$-vectors). However $u A B=0$ also and so $u A$, being in the null space of $B$, has the form $u A=\lambda u$.

We wish to show that $\lambda$ is a linear function of $x_{1}, x_{2}, \ldots$. Suppose that this is not so and put $u A=\left[q_{1}, q_{2}, \ldots, q_{r}\right], u=\left[u_{1}, u_{2}, \ldots, u_{r}\right]$. Then each $q_{i}$ is quadratic in $x_{1}, x_{2}, \ldots$, each $u_{i}$ is linear and we have $q_{i}=\lambda u_{i}$. Suppose $u_{i}$ and $u_{j}$ are non-zero. Since $q_{i} u_{j}=q_{j} u_{i}$ and, by assumption, $u_{i}$ does not divide $q_{i}$, we must have $u_{i}$ and $u_{j}$ are constant multiples of one another. Thus every $u_{i}$ is a constant multiple of some non-zero linear function $\bar{u}$ and consequently there exists a constant non-singular matrix $Q$ such that $X Q$ has the form

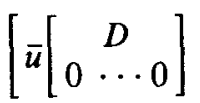

But then rank $D \leqslant r-1$ and this remains true for every specialisation of $X$ to a matrix of $\mathfrak{X}$; thus $\mathfrak{X}$ is not primitive. This contradiction proves that $\lambda$ is linear.

Now put $v=v\left(x_{1}, x_{2}, \ldots\right)=[u,-\lambda]$ an $(r+1)$-vector with linear components and observe that

$$
v X=[u,-\lambda]\left[\begin{array}{cc}
A & B \\
u & 0
\end{array}\right]=[u A-\lambda u, u B]=0 .
$$

Because $v$ is linear the mapping $\theta: \mathscr{X} \rightarrow U$, where $U$ is the space of all row $(r+1)$-vectors, defined by the rule

$$
\sum \alpha_{i} A_{i} \rightarrow v\left(\alpha_{1}, \alpha_{2}, \ldots\right)
$$

is a linear mapping. By specialising the equation $v X=0$ we obtain $\theta(A) A=0$ for all $A \in \mathcal{X}$.

We claim that $\theta$ is one-to-one and onto. Note first that whenever rank $A=r$ we necessarily have $\theta(A) \neq 0$. For suppose, to the contrary, that $\theta(A)=0$ and let $B$ be an arbitrary matrix of $\mathscr{X}$. Then $0=\theta(B) B$ and $0=[\theta(A+B)](A+B)=$ $\theta(B) A+\theta(B) B$ together give $\theta(B) A=0$. Thus the image of $\theta$ is contained in the kernel of $A$ and hence the image of $\theta$ is 1-dimensional. But this means that every matrix of $\mathcal{X}$ has a fixed vector in its null space contradicting the primitivity of $\mathscr{X}$.

We now show that $\theta$ is onto. We have just proved that, for all $A \in \mathscr{X}$ of rank $r$, $\operatorname{ker} A=\theta(\langle A\rangle) \subseteq \operatorname{Im} \theta$. By requirement (iii)' in the definition of primitivity we have

$$
U=\langle\operatorname{ker} A: A \in \mathcal{X} \text { and } \operatorname{rank} A=r\rangle \subseteq \operatorname{Im} \theta .
$$

Finally $\theta$ is one-to-one. Suppose that $\theta(A)=0$ for some $A \in \mathscr{X}$. Then by the argument given above $\theta(B) A=0$ for all $B \in \mathcal{X}$. Thus the entire image of $\theta$ is contained in the kernel of $A$ and, since $\theta$ is onto, $A=0$. 
Let $V$ be the space of $n$-vectors containing the images of $U$ under the matrices

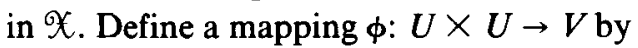

$$
\phi\left(u_{1}, u_{2}\right)=u_{2}\left(\theta^{-1}\left(u_{1}\right)\right) \text { for all } u_{1}, u_{2} \in U .
$$

Obviously $\phi$ is bilinear and, since $u_{1}\left(\theta^{-1}\left(u_{1}\right)\right)=0, \phi$ is also alternating. From the construction it is evident that $\mathscr{X}$ can be identified with a matrix representation of $S(\phi)$ and this completes the proof.

\section{Low rank spaces}

The results of the previous sections allow primitive spaces of matrices of rank at most 2 to be classified immediately. From Theorem A such a space must consist of $3 \times 3$ matrices. Theorem $B$ then shows that the space must be a matrix representation of a space $S(\phi)$, where $\phi$ is an alternating bilinear mapping on $U \times U$ onto $V$ ( $U$ and $V$ both being 3 dimensional). Modulo automorphisms of $U$ and $V \phi$ is unique and one can choose bases of $U$ and $V$ so that $S(\phi)$ is represented by the space of all $3 \times 3$ skew-symmetric matrices.

The classification of primitive spaces of matrices of rank at most 3 presents only slightly more difficulty and we have the following theorem which extends a result of Lloyd (1980).

THEOREM C. Let $\mathcal{X}$ be a primitive space of matrices with $\rho(\mathcal{X})=3$. Then one of $\mathcal{X}$ and $\mathcal{X}^{T}$ (the space of transposed matrices) has the form $S(\phi)$ where $\phi$ : $U \times U \rightarrow V$ is an alternating bilinear mapping, $\operatorname{dim} U=4$ and $\operatorname{dim} V=4,5$ or 6 .

Proof. Theorem A shows that $\mathscr{X}$ is a space of $m \times n$ matrices where one of $m$ and $n$ is 4 and the other is 4,5 or 6 . By replacing $\mathcal{X}$ with $\mathcal{X}^{T}$ if necessary we may take $m=4$. The cases $n=5,6$ are then handled by Theorem B and it is only when $m=n=4$ that additional argument is required.

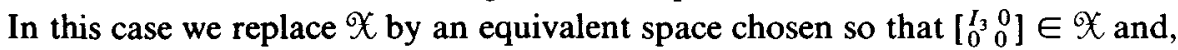
as in the proof of Theorem B, let

$$
X=\left[\begin{array}{ll}
A & v \\
u & 0
\end{array}\right]=\sum x_{i} A_{i}
$$

be a generic matrix (that is, $A_{1}, A_{2}, \ldots$ is a basis for $\mathcal{X}$ and $x_{1}, x_{2}, \ldots$ are independent transcendentals). The equations $u A^{k} v=0, k=0,1,2, \ldots$, may be recast in the form

$$
\left[\begin{array}{c}
u \\
u A \\
u A^{2} \\
\vdots
\end{array}\right]\left[V, A v, A^{2} v, \ldots\right]=0
$$


It then follows (from Sylvester's inequality, for example) that

$$
\operatorname{rank}\left[\begin{array}{c}
u \\
u A \\
\vdots
\end{array}\right]+\operatorname{rank}[v, A v, \ldots] \leqslant 3
$$

Replacing $\mathfrak{X}$ by $\mathfrak{X}^{T}$ if necessary we may take

$$
\operatorname{rank}\left[\begin{array}{c}
u \\
u A \\
\vdots
\end{array}\right]=1
$$

and so $u A \in\langle u\rangle$, say $u A=\lambda u$. This equation also occurs in the proof of Theorem $B$ and we complete the proof of Theorem $C$ by repeating the argument of Theorem B from that point on.

In order to obtain an explicit list of all the inequivalent primitive spaces of matrices with $\rho(\mathfrak{X})=3$ it would be sufficient, in view of Theorem $\mathrm{C}$, to determine all the alternating bilinear mappings from $U \times U$ into $V$ (whose image spans $V$ ) when $\operatorname{dim} U=4$ and $\operatorname{dim} V=4,5$ or 6 . Such a determination (at least for $\operatorname{dim} V=4$ ) depends upon the ground field and is easiest to obtain when the field is algebraically closed. In this case the different mappings have been listed, in characteristics different from 2, by Gauger (1973), see Section 5 and Theorem 7.12. They can be described by the linear dependence relations which hold between the vectors $\phi\left(e_{i}, e_{j}\right)$, where $e_{1}, e_{2}, e_{3}, e_{4}$ is a basis for $U$. The possibilities are

(a) $\operatorname{dim} V=6$ and no dependence relations (other than those implied by $\phi\left(e_{i}, e_{i}\right)=0$ and bilinearity),

(b) $\operatorname{dim} V=5$ and $\phi\left(e_{1}, e_{2}\right)+\phi\left(e_{3}, e_{4}\right)=0$,

(c) $\operatorname{dim} V=5$ and $\phi\left(e_{1}, e_{2}\right)=0$,

(d) $\operatorname{dim} V=4$ and $\phi\left(e_{1}, e_{2}\right)=\phi\left(e_{3}, e_{4}\right)=0$,

(e) $\operatorname{dim} V=4$ and $\phi\left(e_{1}, e_{2}\right)=\phi\left(e_{1}, e_{4}\right)+\phi\left(e_{2}, e_{3}\right)=0$,

(f) $\operatorname{dim} V=4$ and $\phi\left(e_{1}, e_{2}\right)=\phi\left(e_{1}, e_{3}\right)=0$.

We may omit the last of these possibilities for it gives rise to a space $S(\phi)$ which is not primitive. The others give inequivalent primitive spaces and, together with the transposed spaces, there are 10 primitive spaces in all with $\rho(\mathcal{X})=3$.

Finally we can obtain a result on $r$-spaces: spaces of matrices all, except for zero, having the same rank $r$. Westwick (1972) divided such spaces into two kinds, those which are essentially decomposable (they satisfy Theorem $A$ with $\mathscr{Y}$ the zero space of $0 \times 0$ matrices) and those which are not. The indecomposable $r$-spaces seem to be less abundant than the decomposable ones. There are no indecomposable 1-spaces. It follows from Theorem $A$ and the remarks at the beginning of this section that, to within equivalence, the space of $3 \times 3$ skew-symmetric matrices is the only indecomposable 2-space. 
Over algebraically closed fields the indecomposable 3-spaces can also be found. The determination of the primitive spaces of matrices of rank at most 3 together with Theorem A provides a description of all spaces of matrices of rank at most 3 . Routine calculations can then be done to find those which are indecomposable and contain no matrix of rank 1 or 2 . There are just 8 possibilities which we can list as follows.

(i) $m=4, n=6$ : space (a) above,

(ii) $m=4, n=5$ : space (b) above,

(iii) $m=4, n=6$ : 3-dimensional subspace of (i),

(iv) $m=4, n=5$ : 3-dimensional subspace of (ii).

the other 4 possibilities are transposes of these.

\section{References}

M. D. Atkinson and S. Lloyd (1980), 'Large spaces of matrices of bounded rank', Quart. J. Math. Oxford Ser. 31, 253-262.

M. D. Atkinson and S. Lloyd (1981), 'Primitive spaces of matrices of bounded rank', J. Austral. Math. Soc. (Ser. A) 30, 473-482.

M. D. Atkinson and R. Westwick, 'Spaces of linear transformations of equal rank', Linear and Multilinear Algebra, to appear.

H. Flanders (1962), 'On spaces of linear transformations of bounded rank', J. London Math. Soc. 37, $10-16$

M. A. Gauger (1973), 'On the classification of metabelian Lie algebras', Trans. Amer. Math. Soc. 179, 293-329.

S. Lloyd (1980), Computation of bilinear forms and canonical forms of tensors ( $\mathrm{Ph} . \mathrm{D}$. thesis, Cardiff).

M. R. Vaughan-Lee (1974), Breadth and commutator subgroups of $p$-groups', J. Algebra 32, 278-285.

R. Westwick (1972), 'Spaces of linear transformations of equal rank', Linear Algebra and Appl. 5, 49-64.

Department of Computing Mathematics

University College

Cardiff, U. K. 